\title{
Vorwort zur 17. Auflage
}

Seit nahezu 50 Jahren erscheint das „Automobiltechnische Handbuch“. Damals auf knapp 300 Seiten wurde das Wissenswerte über das Kraftfahrzeug gesagt und gezeigt. Heute ist schon durch den Umfang des Werkes und der großen Zahl seiner Abschnitte dargetan, welch ungeheuren Fortschritt die Entwicklung des Kraftfahrzeuges in diesem halben Jahrhundert gemacht hat.

Nach dem zweiten Weltkrieg kam die stürmische Weiterentwicklung des Kraftfahrzeuges. Hier sei nur auf den hohen Entwicklungsstand der Motoren und ihre Leistungssteigerung, auf automatische Getriebe, Gasturbinen, Zubehör und die Schmiermittel hingewiesen. Deshalb war eine vollständige Neubearbeitung aller Abschnitte neben der Einfügung von neuen erforderlich. Alle Neuerungen auf allen Gebieten, die mit dem Kraftfahrzeug zusammenhängen, sowohl die im Inland wie die im Ausland, werden gründlichst behandelt.

Die Gedanken- und Konstruktionsarbeit bei der Neu- oder Weiterentwicklung von Kraftfahrzeugen erfordert weniger materiellen und geldlichen Aufwand als der rein praktische Versuch zum Herausschälen des Guten. $\mathrm{Zu}$ diesen vorbereitenden Gedanken und der Konstruktionsarbeit soll dieses Buch Unterstützung sein.

Ein großer Teil der bisherigen Mitarbeiter konnte wiederum gewonnen werden. Neue hervorragende Kenner ihrer Spezialgebiete kamen hinzu. Ein Mitarbeiter, Herr Dipl.-Ing. Johannes Dony, ist leider durch den Tod aus der Bearbeitung seines Abschnittes „Normung" gerissen worden.

Allen Mitarbeitern und allen Werken, die durch ihre Hilfe das Herausbringen des Werkes bereitwillig ermöglichten, sei an dieser Stelle besonders gedankt.

Leverkusen, Mai 1953

Richard Bussien 
